THEORETICAL ASPECTS 


\section{MODELS OF SPOTS AND FLARES}

D.J. Mu1lan

Barto1 Research Foundation, University of Delaware Newark, Delaware 19711 USA

\section{INTRODUCTION}

MHD effects in stars are seen in their most spectacular form in the processes which are typical of flares. At first sight, it appears that the phenomena of dark spots (whose long. lifetimes give an impression of quasi-equilibrium) are inevitably less interesting. However, this is not necessarily true. Laboratory experiments in recent years have shown that there are many more ways to drive a plasma out of equilibrium than to preserve equilibrium. In that sense, then, it is perhaps "easier to understand" why flares should occur in a stellar atmosphere (where convective jostling of field lines creates potential for driving a large number of instabilities) than why a long-lived feature such as a dark spot should persist. Various instabilities which may contribute to flares are discussed by Priest and Spicer (this volume). Here, we summarize work on the equilibrium structure of cool spots in the sun and stars. Since spots involve complex interactions between convective flows and magnetic fields, we need to refer to observations for help in identifying the dominant processes which should enter into the modelling. This summary therefore begins by discussing certain relevant properties of spots in the solar atmosphere.

\section{OBSERVED CHARACTERISTICS OF SUNSPOTS}

A sunspot is a dark magnetized area of photosphere, with dimensions ranging from $\sim 10^{3} \mathrm{~km}$ up to several times $10^{4} \mathrm{~km}$. Small spots (pores) are uniformly dark, but larger spots are composed of umbra and penumbra. The former is uniformly dark in many cases, but the penumbra is in all cases highly structured, typically filamentary. Penumbral filaments are bright and dark, and they radiate from the umbra towards the undisturbed photosphere. Umbral fields are mainly vertical, but fields are almost horizontal at the outer edge of the penumbra, with a smooth transition in between. Umbral fields are typically 2-4 kG, essentially independent of area.

The visible radiation from an umbra has effective temperature $24000 \mathrm{~K}$, 527 
with surprisingly small deviations from this mean value for spots of widely different areas. Only a small fraction ( $2 \%$ ) of the umbral thermal flux is carried by convection at $\tau_{i}=1$. (Subscript $i$ refers to quantities inside the spot.) This represents a striking reduction in the efficiency of convective transport relative to the photospheric convection, where at $\tau_{e}=1$, convection carries $\sim 15 \%$ of the total flux (Beckers, 1977; Stellmacher and Wiehr, 1976). (Subscript e denotes quantities external to the flux tube.) The missing flux, in the case of a spot occupying an area of 500 millionths of the hemisphere $\left(A=10^{19} \mathrm{~cm}^{2}\right)$ is about $5 \times 10^{29} \mathrm{ergs} / \mathrm{s}$, i.e. $20.01 \% \mathrm{~L}(\mathrm{sun})$. The sun apparently stores this missing flux, or converts it into non-thermal form, in the case of spots which live for more than about 7 days (Willson et a1., 1981). Although this missing flux is small compared to the solar luminosity, it is very large compared to the non-thermal energy flux which is responsible for heating the chromosphere/corona over the spot. Hence, if even a small fraction of the missing flux could be tapped, large flares could be energized above a spot.

A spot appears initially as an enlarged darkening of intergranular material. When the scale size becomes comparable to one granule diameter $\left(\sim 10^{3}\right) \mathrm{km}$, the darkening becomes definitely pronounced: this is a "pore". Most pores survive for only a short time ( $l \mathrm{hr}$ ), and then fade away. Those which survive somewhat longer than this appear to grow by coalescing with other pores. However, even larger pores do not survive long unless they develop a penumbra. Spots which survive for periods of days or weeks in all cases possess penumbrae.

Groups of spots are formed when a large magnetic flux rope erupts through the surface from below. The overall structure is bipolar, but there is usually a pronounced asymmetry between leading and following parts of the bipolar pair: leader spots are usually large and single, whereas the following flux is distributed over an irregular group of smaller spots and pores. The longest-1ived member of a group is usually the leader. Spots which are especially long-lived appear to be surrounded by an organized flow pattern which emerges from the edge of the penumbra and radiates to distances of $210^{4} \mathrm{~km}$. This flow, which sweeps the surrounding area clean of magnetic flux for a time, is called à "moat" (Pardon et al., 1979). Spots decay when separate strands of flux break away (or are torn away) from the main flux rope, and migrate elsewhere. The lifetime of individual flux strands is difficult to estimate empirically, partly because when the strands become small enough to be filled in by radiation, they are difficult to detect. The lifetimes might be as long as a year or more, without contradicting empirical data (Zwaan, 1978). The question of lifetime is an important one in deciding whether the birth of a new active region necessarily indicates the creation of new flux, or whether it may simply represent a re-concentration of already existing (but dispersed) flux.

Flux tubes exist in a variety of forms ranging from large dark spots down to small (sub-arcsecond) bright points in faculae. These 
forms appear to belong to a single-parameter family. The parameter, magnetic flux $\Phi$, is smaller than $\sim 10^{19}$ maxwells in small flux tubes which appear bright at all altitudes in the photosphere, whereas for $\Phi \geq 10^{19}$ maxwe11s, the flux tube appears dark, at least in the photosphere (Frazier, 1977). We note that, since photospheric fields are in almost all cases of order 1-2 kG (Stenf1o, 1973), a critical flux of $10^{19}$ maxwells corresponds to a critical length scale of order $10^{3} \mathrm{~km}$, i.e. about one granule diameter. Thus, interference between magnetic fields and convection is central to the phenomenon of dark spots on the sun.

The flux which emerges through an umbra is only part of the total flux in an active region. Surrounding the spots, magnetic fields exist throughout the active region, and these cause not a darkening, but a brightening in the chromosphere. (We refer to this generically as "plage".) In plage, the fields are not organized into clearly identifiable large-scale entities: "cell"-structure is very irregular, apparently indicating that the fields in plage are generally more random in direction than in spots. (Randomness of the fields contributes to chromospheric heating, whether one considers heating due to wave dissipation or reconnection.) This suggests that we might usefully consider the following definition for the distinction between spotted and non-spotted (plage) areas of an active region: in a spot, the field is (somehow) organized into an entity of vertical flux which is coherent over a horizontal scale in excess of one granule diameter. Plage represents "everything else" in the active region. The ratio of spotted area to plage area in an active region is difficult to extract from the literature. It clearly depends on the age of the active region: in an old active region, plage persists even after spots have disappeared, whereas in the young phase, spots are the pre-eminent characteristic of the active region at the photospheric leve1. In the context of the proposed definition, the penumbra should be classified as "plage". Thus, a pore represents the limit of large spot/plage ratio.

Although the photospheric level of an umbra remains darker than normal for prolonged times, this does not mean that time-dependent phenomena are entirely absent from the umbra. On the contrary, umbrae and penumbrae are observed to be sites of a rich variety of dynamic phenomena, although the energy transport associated with these is only a small fraction of the missing flux. When an umbra is observed in chromospheric radiation, transient brightenings, waves, and various oscillations (with periods ranging from 100-500 seconds and longer) are detected (Moore, 1981). The ultimate manifestation of energetic processes in an umbral flux tube occurs during certain very large flares: in "proton flares", the umbral flux tube emits enhanced chromospheric radiation, suggesting that the umbral chromosphere has been forced down to lower altitudes. De Jager (1968) suggested that when large flares occur close together in time in a particular active region, the most likely source of energy is the missing flux in spots in that active region: no other source of energization seems 
adequate. However, De Jager did not specify how the missing flux might be tapped in order to power the large flares.

\section{THEORE TICAL STUDIES OF SPOTS: SUMMARY}

of the several topics which arise in connection with theoretical studies of spots on the sun and other stars, we select four for comment in the present paper: field concentration, stability, cooling and missing flux, and relationship with flares. In each case, we will discuss the topic initially with reference to the sun, and then go on to consider implications for spots on cool dwarfs. In the case of starspots, the primary aim of theoretical work at the present time (in view of the available observational data) should be the prediction of the areas of starspots on stars of various spectral types, the effective temperatures of the starspots, and the ratio of spot area to plage area in a stellar active region. Unfortunately, as will become clear in the discussion, none of these quantities can be predicted with great confidence at the current level of our knowledge. Thus, it appears likely that observations will lead theory in the starspot problem for some years to come.

\section{CONCENTRATION OF MAGNETIC FIELDS}

During the growth phase of large sunspots, the magnetic flux in the spot grows so rapidly that it cannot be the result of concentration of weak flux already in the photosphere by supergranule velocities (Zwaan, 1978): in order for this to be the case, some 35 supergranules would have to be "swept clean" and concentrated into one region, and the velocity of the flow would need to be $1 \mathrm{~km} / \mathrm{s}$. The velocity is too high to be consistent with observed supergranule velocities, and it seems improbable that a coherent convergent flow would arise embracing 35 supergranules at one time. Hence, the flux rope which emerges in the form of a sunspot must have al ready been concentrated at depth, prior to eruption (Piddington, 1976). It is not possible at present to estimate how strong the magnetic field might be at great depth in the convection zone. However, Zwaan (1978) suggested that equipartition with turbulent velocities might be appropriate: this leads to about 10 $\mathrm{kG}$ at depths of order $10^{5} \mathrm{~km}$. Near the surface, the equipartition field strength is found to be about $600 \mathrm{G}$. Although there is some evidence that flux does emerge at the solar surface with fields of this order, subsequent strengthening of the field apparently occurs, since the flux tubes which are most common in the photosphere at any one time appear to have fields appreciably stronger than the above value: typical strengths are $1.5-2 \mathrm{kG}$. The cause of the extra strengthening is probably related to surface cooling of the flux tube: with cool gas inside the flux tube, the pressure scale height becomes less than that outside, and the internal gas slumps to lower levels than the external gas. At any particular geometric depth, then, the external pressure exceeds the internal pressure, and the effect is to compress the flux tube. Thus, field concentration may have two separate components, one operating at depth, the other operating near the surface where cooling 
is permitted.

According to this view, pores and individual flux tubes in an active region on the sun's surface can be considered as separate strands of flux belonging to the main flux rope which creates the active region as a whole. The strands are thought to be attached to the main rope somewhere beneath the surface. This view explains why pores appear to coalesce into a single spot on the surface: what one is watching on the surface is a two-dimensional "cut" across a three-dimensional "rising tree" of flux (Vrabec, 1974), in which separate strands of flux correspond to the "branches", while the emergence of the main "trunk" of the tree creates a large umbra with the appearance that all of the branches have been "swept" into the large umbra. The sweeping of pores into a single umbra is therefore only a projection effect, rather than being evidence for surface motions acting to push magnetic strands together.

The creation of magnetic flux at great depths in the convection zone of a star (or below the convection zone) is perhaps due to dynamo action (Belvedere, this volume). Hence, if we wish to predict fluxes in active regions in cool dwarfs, it is necessary to know the physical conditions at great depth. In the limiting case of low mass stars, this means, in effect, knowing the physical conditions essentially all the way to the center of the star. Unfortunately, there are at present serious uncertainties in such basic quantities as the depth of the convection zone, the convective efficiency, the turbulence velocities etc., in models of low mass stars (Cox et a1., 1981). Thus, by changing the opacities near the surface to include previously neglected contributors, Cox et a1. (1981) found that a star which used to be believed to be fully convective may instead possess an extensive radiative core. Hence, application of various scaling laws to the various internal physical variables along the lower main sequence (in order to predict dynamo properties) must be considered as quite uncertain at present.

The area $A_{S}$ of a flux rope on the surface of a star is determined by the total flux, $\Phi$, and the surface field strength, $B_{S}$ : $A_{S}=\Phi / B_{S}$. The value of $B_{S}$ is observed to be remarkably uniform in the sun: from the largest spot down to the smallest detectable facular bright point, the value of $B_{S}$ is within a factor of 2 of $2 \mathrm{kG}$. Within the framework of the ideas mentioned above concerning the slumping of gas within a flux tube, we denote by $D$ the depression of the layer $\tau_{i}=1$ (i.e. optical depth unity inside the flux tube, where pressure is $p_{i}$ ) below the layer $\tau_{e}=1$ ( $i . e$. optical depth unity in the photosphere). At depth $D$ below $\tau_{e}=1$, we can write $\mathrm{pe}_{\mathrm{e}}(\mathrm{D})=\mathrm{p}_{\mathbf{i}}+\mathrm{B}^{2} / 8 \pi$, if the $\mathrm{flux}$ tube is assumed to be in magnetohydrostatic equilibrium (MHSE). In the limit of total evacuation of the flux tube, the value of $B_{S}$ can be approximated by $\left(8 \pi p_{e}(D)\right)^{1 / 2}$. For example, to reproduce a surface field of, say, $4 \mathrm{kG}$, as is observed in some large sunspots, this relation requires us to pick D $400 \mathrm{~km}$ (according to the convection zone model of Spruit, 1977). Finite $\mathrm{p}_{i}$ 
increases D. Weaker fields require smaller values of $D$, the Wilson depression. The fact that flux tubes at different parts of the sun are observed to have more or less the same values of $B_{s}$ reflects the condition that on a large scale, the solar photosphere is spherically symmetric.

If this is a correct interpretation of the properties of flux tubes near a stellar surface, then evaluation of $B_{S}$ in red dwarfs will require a knowledge of the depth-dependent pressure, as well as an estimate of the appropriate Wilson depression. When we recall that in a star such as UV Ceti, where photospheric temperatures are of order $2600 \mathrm{~K}$, and gravity is of order $10^{5} \mathrm{~cm}^{2} / \mathrm{s}$, the pressure scale height is only about $10 \mathrm{~km}$, it is clear that a small error in estimating $D$ will result in a large uncertainty in $P_{e}(D)$, and therefore in estimating $B_{S}$ in such a star. The serious uncertainties associated with choice of opacity in the outer layers of cool dwarfs, as well as with choice of the adiabatic gradient (due to molecule formation) have been stressed by Bohn (this volume). Even if both of these variables were well known, however, there remains the long standing uncertainty in the treatment of stellar convection, particularly in the near-surface layers where the degree of superadiabaticity is most pronounced.

Thus, prediction of starspot area $A_{S}$ from first principles requires knowledge of physical conditions not only near the base of the convection zone but also near the top. In view of these difficulties, it is not surprising that simple prescriptions have occasionally been used to "predict" spot sizes. Supergranules were considered important elements in the prescriptions proposed by Mullan (1973) and Rucinski (1979). However, as the importance ascribed to supergranule flows in concentrating spot fields has diminished over the years (Zwaan, 1978), the arguments based on supergranule properties seem to loose much of their forcefulness. In fact, it now seems more likely that supergranule sizes and flow patterns may be controlled by the magnetic field, rather than the reverse, at least in the regions of primary interest to us here (i.e. regions of strong B)(cf. Zwaan, 1978). For example, the only place where the supergranule flow is "permitted" to organize itself into a large annular cell appears to be in vicinity of a well developed sunspot; and in an active region, the supergranule cellular pattern is not "permitted" to be established, and the network is quite chaotic in character in an active region.

\section{STABILITY OF SPOTS}

The one-parameter family of magnetic flux elements has been interpreted by Spruit (1977) in terms of a model of flux tubes in MHSE. In this model, a vertical flux tube is supposed to be the site of a reduced upward flux of thermal energy $\left(F_{i}<F_{e}\right)$ relative to the external undisturbed photosphere, where the upward flux is $F_{e}=\sigma T_{e}^{4}$ $\left(\mathrm{T}_{\mathrm{e}}\right.$ is the solar effective temperature). The reduction in flux inside the flux tube is presumably related to the reduction 
of convective efficiency by the vertical magnetic field, although the details of the origin of $\mathrm{F}_{i}<\mathrm{F}_{\mathrm{e}}$ are not crucial for Spruit's models. The flux tube is also characterized by lower temperatures, $\mathrm{T}_{i}<\mathrm{T}_{e}$. Again, the specification of the cooling agent is not crucial (cf. also Low, 1980). Influx of radiation from the walls of the tube reduces the difference $\mathrm{T}_{e}-\mathrm{T}_{\mathbf{i}}$ in the surface layers, at least in the case of small tubes (with diameters less than about $10^{3}$ $\mathrm{km}$ ). Larger tubes, such as spots, do suffer somewhat from lateral influx of radiation, but not enough to compensate for the darkness of the spot due to reduced $F_{i}$. Spruit showed that as the flux tube diameter increases from values much less than $10^{3} \mathrm{~km}$ to larger than that, there is a smooth progression from bright points (such as faculae) to dark spots; in good agreement with the one-parameter family discovered by Frazier (1977) in the sun.

The question which arises in connection with equilibrium models of flux tubes now is the following: are the equilibria stable? In general, the answer is no. A vertical flux tube is a region in which the magnetic field is attempting to exclude the external, higher pressure gas from entering. This situation, which arises in plasma fusion devices such as bottles and various pinches, is known to be unstable to interchange and flute instabilities (Spitzer, 1962; Priest, this volume). Thus, in the case of a bundle of vertical flux strands surrounded by field free gas, if one interchanges one of the flux strands with a column of field free gas from outside the bundle, the total potential energy of the system can be reduced. As a result, a bundle such as a spot should shred into separate strands in a time scale which is essentially the Alfven wave crossing time, $R / v_{A} \sim l$ hour in the case of spots on the sun. The fact that many spots survive for periods of time which exceed this disruption time-scale by several orders of magnitude indicates that powerful stabilizing forces of some kind are at work to hold the spot together. We can discuss the stabilizing forces most conveniently by artificially separating the discussion of surface layers from the discussion of the layers at great depth below the surface.

To stabilize the surface layers, Meyer et al. (1977) proposed to use buoyancy forces. Suppose the flux tube, instead of being vertical, fanned out in some region so that the strands of flux were inclined to the vertical, and extended out from the main flux rope over the top of the non-magnetic gas outside the rope. Because of MHSE, the gas inside the strands is of lower density than the field-free gas. Now consider the process of attempting to interchange a flux strand inside the rope with some field-free gas fram outside. Since the field-free gas lies deeper than the overriding strands which fan out from the rope, work must be done to raise the field free gas up to the level where the strand of flux is. And since the density inside is less than density outside, this requires more work to be done against gravity than is released when the flux strand completes the interchange by sinking into the field-free gas. As a result, buoyancy can convert the equilibrium into a stable one, provided that the inclination of the fanning-outflux 
strands to the vertical is larger than a critical value. Now fanning out is precisely the characteristic which most closely describes penumbral structure, and it is interesting that all spots which survive for periods of time of, say, a day or more possess penumbrae. It seems that the formation of a penumbra signals the spot's attempt to stabilize itself, at least in the surface layers.

The requirement of a critical amount of fanning out of field lines is necessary in order that buoyancy forces can operate during an attempted interchange. Now, in MHSE, the cause of fanning is a rapid drop in external pressure. Suppose, however, that a spot is so small that its radius $R$ is less than the pressure scale height, $H_{p}$, in the external gas. Then over a height range of order $R$, the pressure does not vary significantly outside the tube, and hence the tube has little incentive to fan out. As a result, Meyer et a1. (1977) concluded that flux tubes could not be stable if they were too small, i.e. if the total amount of flux fell below a certain limit. Using empirical solar models, Meyer et al. found that the critical flux which is required for surface stability is ${ }^{1} 0^{19}$ maxwells. This is in remarkably good agreement with the empirical boundary between dark and bright spots. (Meyer et al. did not consider lateral influx of radiation, and therefore did not deal with bright flux tubes, as Spruit (1977) did.)

To stabilize a spot at great depth requires a different mechanism. There, the flux tube is expected to become essentially vertical, or perhaps even fan out again. In either case, buoyancy forces cannot be called upon to stabilize the structure at depth. What then can combat instability? Meyer et a1. (1974) proposed that converging flow due to reversed supergranule flow at depths $\mathrm{d}_{\mathrm{C}} \sim 2 \mathrm{R}$ might serve as a "collar" to hold the flux rope together. They proposed that the convergent flow, when it encounters the wall of the tube, flows upwards, and eventually creates the outward flow around the surface layers of the spot which is observed as the "moat" around long-lived spots. This is consistent, therefore, with the observation that spots with moats are the most stable spots, in general. Since a moat is a three-dimensional annular flow which is organized coherently over scale sizes of order $\mathrm{d}_{\mathrm{c}}$ (i.e. several times $10^{4} \mathrm{~km}$, in the case of the largest spots), the flux tube beneath the surface of the spot must remain more or less vertical over depths of order $d_{c}$ if moat formation is to be permitted. A non-vertical flux tube is not conducive to setting up and preserving the 3-D annular flow pattern of a moat. This has an important implication for differences between leading and following spots in the sun, where the angular velocity of rotation, $\Omega$, increases inwards (as various data suggest). Meyer et al. (1977) pointed out that because $d \Omega / d r<0$, the flux rope beneath a leader spot is more likely to be vertical than the flux rope beneath a follower spot in the sun. Thus, moat formation would be easier around the leader, and this could explain why leaders usually are the longest-lived spots. Moreover, in the case of the follower spot, not only is the flux tube non-vertical, but the field lines are strongly curved in the surface layers on the side of the follower spot which lies closest to 
the leader. As a result, curvature forces are more severe on the field lines in the follower spot, and flux tends to shred away from the follower. This would explain why follower flux tends to be distributed among many small spots and pores.

What are the implications of these ideas for starspots? The most prominent characteristic of active stars is fast rotation. This is especially true of stars which are observed to have cool spots on their surface: stars which rotate with periods longer than about 7 days tend to show strong emission in, say, Ca K, i.e. they are "plage" stars, whereas stars which rotate with periods shorter than 7 days are spotted stars (Vogt, this volume). If we accept the definition proposed above for the distinction between spotted and plage areas of an active region, then we conclude that fast rotation apparently encourages the appearance of flux ropes which are primarily vertical at the stellar surface and coherent over length scales of at least one granule diameter. Slower rotation may also create magnetic flux, but apparently it intersects the surface in a more chaotic manner, or else the flux in individual tubes is simply not large enough to engulf a single granule (given the constraints of surface field strength imposed by the photospheric pressure distribution). However, given the fact that faster rotation favors creation of spots, what does the faster rotation do to the stability of those spots? It seems plausible that as $\Omega$ increases, $|\mathrm{d} \Omega / \mathrm{d} r|$ should also increase. If this is true (and it must be admitted that high turbulent viscosity in convection zones would generally be expected to keep $|\mathrm{d} \Omega / \mathrm{d} \mathbf{r}|$ small: quantitative evaluation will ultimately be required), it leads us to the following conclusion: when a flux rope erupts to the surface of a spotted star, the rope will be sheared beneath the surface more seriously than in the solar case. Therefore, even the leader spot in a bipolar pair might have its flux tube tilted seriously away from the vertical beneath the surface (and the follower more so). As a result, a moat could not form even around the leader spot. This argument leads us to the conclusion that large stable spots may not exist on the surfaces of fast rotating active stars. Instead, the flux rope is expected to break up into many small spots, each of which is in a stage of transient evolution, breaking away from the main flux rope. Thus, spotted stars would not possess analogs of what is perhaps the most prominent hall-mark of solar activity: large, single long-lived more-or-less symmetric spots. Ideally, the presence of multiple spots on stellar surface can be proven by careful photometry of an eclipsing system during ingress or egress. However, the expected amplitudes of fluctuations from individual spots may be too small to be detectable, even with the best available photometers (Caton, 1981).

A further conclusion can be drawn from the necessity of many small spots (rather than a single large spot). Small spots are, as we have mentioned, less likely to form penumbrae. Spot groups on active stars may therefore be characterized by larger numbers of umbrae, without as much penumbral coverage as in the sun. In such a case, the spot/plage ratiowould be larger than in the solar atmosphere. of 
course, these arguments will remain speculative until more is known about $\mathrm{d} \Omega / \mathrm{dr}$ in cool dwarfs.

So far, we have mentioned the proposal of Meyer et al. (1974) to stabilize spots at depth by means of moat flow. Now, most spots are not observed to be surrounded by moats. In the absence of any other stabilizing influence (such as strong twists), most spots may not have an appropriate "collar" at depth, and should therefore not be stable. This led Parker (1979) to propose that indeed a spot flux rope succumbs to instability beneath the surface (below 1-2 thousand $\mathrm{km}$ ), and filaments into separate flux strands, with field-free gas surrounding each separate strand. In Parker's view, a spot is a dynamical clustering of separate tubes, and the spot will disperse as soon as the clustering force disappears. As a source for the clustering force, Parker proposed a converging flow (as Meyer et al. did), but instead of the flow turning upwards along the walls of the tube, Parker proposed that the flow went downwards. (This helps then not only with the stability problem, but also with the cooling problem: see below). Evidence for large-scale organized sub-surface flows persisting for days or weeks around spots is weak at present. However, Parker's suggestion explains why spots of very different areas can have properties (such as effective temperature and magnetic field strengths) which are remarkably uniform: these properties are determined by the elementary flux tubes themselves, and different spots differ only in total numbers of elementary flux tubes which they contain.

\section{SPOT COOLING AND MISSING FLUX}

So far, we have said nothing about thermal equilibrium of flux tubes. Models of flux tubes in MHSE have simply assumed that the thermal flux inside the tube is reduced somehow, without discussing the energy equation. In fact, it is by no means obvious that the constraints of thermal equilibrium can be satisfied within the context of some of the MHSE models which have been constructed (Low 1980). Zwaan (1978) has summarized the topic of spot cooling succinctly: "the heat balance is the ultimate problem in long-lasting flux tubes." The missing flux is certainly not present in thermal form (Willson et al., 1981): when spots are present on the surface of the sun for at least 7 days, the solar power output is reduced by essentially the spot missing flux. It is true that some compensation for the spots may be provided by facular emission, but this compensation is not total. The missing flux may be emerging from the sun in non-thermal form (thereby escaping detection by the radiometer used by Willson et al.), or else it may be stored inside the sun. A recent theoretical discussion by Spruit (1982) on the time-dependent heat transfer problem which arises when a cool spot appears on a stellar surface has shown that almost all of the missing flux can be stored inside the sun for long time scales, if necessary (longer than a year).

The question of storage of missing flux of starspots inside the star is in principle susceptible to observational testing: what is 
necessary is bolometric luminosities at times when a spot is present on the surface and when it is absent. In view of the low effective temperatures of red dwarfs, this requires photometry out to wavelengths of at least several microns. As far as I know, such measurements have not been made for any red dwarfs. The simpler problem of searching for a "bright ring" around starspots has, to the best of my knowledge, also not been attacked. Historically, prior to the data of Willson et al., it was always possible to say that the sunspot missing flux was simply re-distributed over a large area of the surrounding photosphere, so large that the enhancement relative to the normal photosphere would be lost in the noise of granulation fluctuations. In fact, Spruit (1977) showed how easy it is for the sun to "hide" the missing flux in this way. However, in a red dwarf, where the missing flux may amount to some tens of percent of the total luminosity, the brightness of the surrounding "undisturbed" photosphere would certainly be enhanced by detectable amounts even if the missing flux were spread out over the entire area of the unspotted photosphere. There are now less pressing reasons to search for bright rings around starspots, since solar data suggest that temporal redistribution of the missing flux is at work, rather than spatial redistribution (see also Hartmann and Rosner, 1979).

Very recently, however, the possibility that the missing flux in starspots is actually emerging into the corona as hydromagnetic waves, and dissipating there, has been proposed by Gershberg (this volume). He has pointed out that the $X$-ray power emitted by flare stars in their quiescent condition is comparable to the missing power in the dark spots on the stars concerned. He interprets this as evidence that a significant fraction of the energy trapped in subphotospheric gas can be transferred effectively into higher regions of a stellar atmosphere by hydromagnetic waves. Our solar prejudices force us to consider this suggestion as a daring one, indeed. It is well known that the corona above an active region emits in X-rays at a level which is orders of magnitude below the level of missing power due to spots in that region (Evans et a1., 1977). And there are theoretical arguments which suggest that Alfven waves coming up from sub-photospheric gas along vertical magnetic field lines are strongly reflected (Thomas, 1978). This has led to the conclusion that if Alfven waves are indeed carrying the missing flux in spots, only a small fraction (of order $10^{-5}$ ) would normally be able to leak upwards into the corona (Mullan, 1981). On the other hand, other studies of the propagation of Alfven waves in the solar atmosphere have shown the possibility of resonances, such that waves of certain preferred periods may encounter much less serious reflection at the temperature minimum. In particular, the propagation characteristics along a coronal arch may be such as to raise the transmission coefficient of certain Alfven waves to the corona by orders of magnitude, thereby allowing those waves to heat the corona strongly along the arch (Zhugzhda, 1982). Loops which emerge from sunspot umbrae are often rather cool (Orra11 1981). Hence, the resonance behavior of loops apparently does not help to improve the transmission of Alfven waves from umbrae to corona in the sun (assuming Alfven waves are present in umbrae). 
If Gershberg's proposal can be validated, it may indicate that conditions in coronal loops which emerge from umbrae of starspots may be more conducive to transmission of MHD waves from beneath the surface of the umbra into the corona. For example, a resonance condition of the kind $\mathrm{P}=\mathrm{L} / \mathrm{v}_{\mathrm{A}}$ (where $\mathrm{P}$ is wave period, $\mathrm{L}$ is loop length, $\mathrm{v}_{\mathrm{A}}=$ Alfven speed) is probably not satisfied in the solar loops, where Ln $10^{9} \mathrm{~cm}, v_{A} \sim 10^{8} \mathrm{~cm} / \mathrm{s}$. In such loops waves would need to have periods of order $10 \mathrm{~s}$ to resonate. However, MHD waves in sunspots are expected to have periods of a convective turnover time, or times of order $10^{2}-10^{3} \mathrm{sec}$ (Mullan, 1981). Application of the same a rguments to red dwarfs, however, suggest that since loop lengths a re in all likelihood longer in such stars (perhaps as large as a stellar radius; Kodaira, this volume), whereas $\mathrm{v}_{\mathrm{A}}$ may not be very different from solar values (Mullan, 1975), the resonant periods may increase to greater than $10^{2}$ seconds. Such periods would lie within the spectrum of periods predicted by convective turnover time-scales (the latter can be extracted from the models of Mullan, 1974b), and it is therefore not excluded that resonant transfer is considerably more efficient in red dwarfs than in the solar case.

The possibility that the missing flux from spots may be used for coronal heating in red dwarfs needs to be explored in some detail. It is characteristic of Alfven waves that they have great dissipation lengths. For this reason, they have traditionally been considered as the favorite wave mode for heating of the corona of the sun (since other modes would have dissipated low down in the chromosphere). However, in the absence of evidence for Alfven waves passing up through the chromosphere, the role of Alfven waves in heating the solar corona has diminished in favor of other mechanisms. Gershberg's suggestion indicates that red dwarf coronae may be tapping a reservoir of mechanical energy which is unimportant in the sun, namely, MHD waves in spots. In view of the long dissipation lengths, tapping of such a reservoir would be expected to enhance coronal heating more than chromospheric heating. This would be a very useful feature, since it has been found empirically that red dwarfs are more efficient at heating their coronae than at heating their chromospheres (Mullan 1979), whereas in the sun, the reverse is true.

How, then, can we estimate how cool a spot should be, and how much missing flux should there be in a starspot? Attempts to estimate spot cooling by prescribing velocity fields are valuable in the case of the sun, where the velocity fields can be observed (Schatten, 1981). However, this approach cannot be carried over to red dwarfs. The question of spot cooling can be discussed consistently only by means of a model for stellar convection. In this regard, discussions of the onset of convection (i.e. in terms of critical Rayleigh numbers) seem to be of rather limited applicability. For example, the criteria derived from linear theories for the magnetic inhibition of convection are too strong to apply to the solar case (Staude, 1978). Even in the presence of a magnetic field of 3-4 kG, solar convection cannot be suppressed completely because the electrical conductivity is far from 
infinite. Thus, from the point of view of modelling, it is natural to consider convection in a spot in terms of the same model as one uses for the undisturbed convection zone, with some modification for the presence of the vertical field. In particular, in a compressible medium, the convective heat flux depends on the local temperature gradient in an essentially non-linear way (including threshold behavior). Failure to appreciate this has led to conclusions about spots which were seriously in error (cf. Spruit, 1977). Moreover, the convection model must include horizontal motions: in a convection model where only vertical flows are calculated, there is no physically meaning ful way to parameterize the reduction of convective efficiency in the presence of a vertical field. Furthermore, the combination of horizontal flows with vertical fields allows the model to include conversion of convective energy to MHD waves mechanically. Thus, the severe thermodynamic and conceptual problems which arise in attempting to discuss a cool spot in terms of a heat engine which emits Alfven waves by means of overstable oscillations (Cowling, 1977) do not arise. Reduction of convective efficiency and copious emission of MHD waves are not mutually exclusive in a sunspot.

A model which incorporates the above requirements was published severa1 years ago (Mullan, 1974a). It led to sunspot effective temperatures of less than $3000 \mathrm{~K}$ in a $3 \mathrm{kG}$ field, and effective temperatures of less than $2000 \mathrm{~K}$ in the case of a spot on a red dwarf (Mullan, 1974b). In the latter case, arguments were presented which suggested that the surface fields in starspots should be larger than in the solar case by an order of magnitude. As far as I know, there are no other predictions of effective temperatures of starspots. Staude (1978) applied a cellular model of convection to the sunspot problem, but no application to starspots was made. In my models, the missing flux was carried by Alfven waves. In the case of sunspots, these are expected to be reflected inside the sun. They will eventually dissipate and return their energy to the thermal pool inside the sun. However, as far as the surface of the sun is concerned, the missing flux will essentially be stored as long as the spots are visible. We can estimate the time-scales for Alfven wave dissipation inside the sun due to ohmic effects, if we select appropriate parameters, e.g. fields of $10^{4} \mathrm{G}$ at depths of $10^{5} \mathrm{~km}$, where $\rho \sim 0.2 \mathrm{gm} / \mathrm{cm}^{3}, T 2 \times 10^{6} \mathrm{~K}$. Then for waves of periods $P=10^{2}-10^{3} \mathrm{sec}$ (typical of sunspots, according to the models), we find dissipation times of 6-600 days. The solar radiometer experiment (Willson et a1., 1981) shows that the solar output shows no fluctuations on time-scales shorter than about 7 days. It appears that storage of sunspot missing flux in the form of Alfven waves inside the sun is not incompatible with the solar radiometer data.

\section{RELATIONSHIP BETWEEN SPOTS AND FLARES}

Most (99\%) solar flares occur in the vicinity of spots. Is the spot-flare relation purely passive (in the sense that both require strong fields), or is it active? In the former case, the major role to be played by spots would be in defining the separatrices across which 
flux transfer would occur during flares (Baum and Bratenah1, 1980). In the latter case, the spot would contribute to flare energization, particularly in flares where storage of energy in the corona is inadequate (Piddington, 1973; Spicer, this volume). The proposal that Alfven waves carry the missing flux of spots, and that these waves are normally trapped beneath the surface, provides a natural context for exploring the possibility of an active spot-flare connection. During large proton flares, the chromosphere/corona is forced downwards over umbrae, thereby enhancing the transmission of Alfven waves (non-resonantly) into the corona. Even if only $1 \%$ of the missing flux reaches the corona, a large fraction of the flare energy budget can be supplied (Mullan, 1981). This is therefore a specific mechanism to implement De Jager's (1968) suggestion that large solar flares may be powered by the missing flux in spots. In the context of stellar flares, the proposal that flares are powered by the missing flux of spots (or a fraction thereof) can be used to predict flare frequency as a function of stellar luminosity with some success over a range of some 10 magnitudes (Mullan, 1975b).

To the extent that there is an active relationship between spots and flares, spots should be viewed therefore not simply as cool areas which are dull, compared to the more interesting behavior exhibited by flares: rather, spots should be viewed as engines which do the work of converting the energy of convective flows into flare-compatible form.

\section{REFERENCES}

Baum, P.J. and Bratenahl, A.: 1980, Solar Phys. 67, 245.

Beckers, J.: 1977, Ap. J. 213, 900.

Caton, D.B.: 1981, Publ. Obs. Appalachian St. Univ., Vol. I, part 2 .

Cowling, T.G.: 1976, Mon. Not. Roy. Astron. Soc. 177, 409.

Cox, A.N. et a1.: 1981, Ap. J. Letters, 245, L37.

DeJager, C.: 1968, in Structure and Evolution of Active Regions, ed. K. Kiepenheuer (Dordrecht: Reide1), p. 480.

Frazier, E.N.: 1977, Astron. AP. 64, 355.

Ha rtmann, L. and Rosner, R.: 1979, Ap. J. 230, 802.

Low, B.C.: 1980, Solar Phys. 67, 57.

Meyer, F. et a1.: 1974, Mon. Not. Roy. Astron. Soc. 169, 35.

Meyer, F. et a1.: 1977, Mon. Not. Roy. Astron. Soc. 179, 741.

Moore, R.L.: 1981, Space Sci. Rev. 28, 387.

Mullan, D.J.: 1973, Ap. J. 186, 1059.

Mullan, D.J.: 1974a, Ap. J. 187, 621.

Mullan, D.J.: 1974b, Ap. J. 192, 149.

Mullan, D.J.: 1975a, Astron. Ap. 40, 41.

Mullan, D.J.: 1975 b, Ap. J. 200, 641.

Mullan, D.J.: 1979, Irish AJ 14, 73.

Mullan, D.J.: 1981, Solar Phys. 70, 381

Orrall, F.Q.: 1981, Space Sci. Rev. 28, 423.

Pardon, L. et a1.: 1979, So1. Phys. 63, 247.

Parker, E.N.: 1979, Ap. J. 230, 905.

Piddington, J.H.: 1973, Solar Phys. 31, 229. 
Piddington, J.H.: 1976, Ap. Space Sci. 40, 73.

Rucinski, S.: 1979, Acta Astron. 29, 203.

Schatten, K.H.: 1981, Ap. J. 247, L139.

Spitzer, L.: 1962, Physics of Fully Ionized Gases (Interscience).

Spruit, H.C.: 1977, dissertation, Utrecht.

Spruit, H.C.: 1982, Astron. Ap. 108, 348.

Staude, J.: 1978, Bul1. Aston. Inst. Czechoslov. 29, 71.

Stellmacher, A. and Weihr, E.: 1976, Astron. Ap. 47, 479.

Stenf1o, J.0.: 1973, Solar Phys. 32, 41.

Thomas, J.H.: 1978, Ap. J. 225, 275.

Vrabec, D.: 1974, in IAU Symo. 56, 201.

Willson, R.C. et a1.: 1981, Science 211, 700 .

Zhugzhda, Y.: 1982, Solar Phys. 81, 245.

Zwaan, C.: 1978, Solar Phys. 60, 213 .

\section{DISCUSSION}

Uchida: The idea of storing energy in the form of Alfvèn waves is interisting but it is possible to store the waves without dissipation down in the high $\beta$ region?

Mullan: Estimates of the time for dissipation of an Alfvèn wave depend critically on the field strength and the density i.e. on the Alvèn speed to a high power. I do not believe that our models of the deep convection zone are yet good enough to provide reasonable estimates of what the value might be. So I cannot estimate the lifetime of an Alfvèn wave below the surface at this moment.

Gibson: I would like to offer some independent evidence for the leading spot hypothesis. When you look at the non-eclipsing RS CVn binaries, $i$. e. the ones whose orbital planes are apparently titled with respect to our line-of-sight showing more of the pole, you expect on the leading spot hypothesis to see more intense magnetic field either coming toward you or going away from you. In the radio data for these stars one sees a particular handedness of circular polarization, e.g. for HR 1099 we consistently see $20 \%$ circular polarization. In the eclipsing systems, where presumably you see equal contributions from the Northern and Southern hemispheres, we see no circular polarization especially at the quiescent times.

Mullan: I wonder whether you can separate, in that case, the effects of local from those of global fields. If you happen to be looking at a non-eclipsing system then one of the poles is tilted toward you. So are you sure that you can distinguish this effect?

Gibson: No, I cannot do that. Buth what I am saying is that the leading spot would presumably have a larger field than the trailing field and 
so, since the non-thermal emission varies as $B^{2}$, the bulk of that emission would be coming from over the leading spot. That is the reason for my comment.

Mullan: I do not think that I said that the field of a leading spot would be larger than that of a following spot. At least, I did not mean to say it. What I meant to say was that the leading spots on the Sun are more stable than those following.

Weiss: It is true that if the following spot broke up into separate flux tubes their field strengths would be small up in the corona.

Spicer: You did not say anything about the possibility of parallel currents flowing in these flux tubes attracting one another. Suppose, for instance, one had a lot of pores and they had parellel currents then they would attract and coalesce. So parallel currents give rise to coalescence instability, to filamentation effects and also to an energy transport mechanism. They also help to stabilize. Neither Spruits' nor Parker's calculations, as well as elsewhere in the literature, make allowance for these currents.

Mullan: Well, the coalescence of pores has been observed but one is not forced to conclude that they are being swept together by the velocity fields. There could be other interpretations. If your suggestion is that parallel currents can sweep pores together then that strengthens the point I was making. That point was that one cannot rely on surface velocity fields to do the coalescence. The problem of currents in spots has not been treated except in one case by Gopelli (?) and Zwaan who believed they could help to stabilize the spot by having a current sheet around the flux tube. There are however some reasons why the numerical values they used might be difficult to justify.

Weiss: I would like to make two quick comment on that. First of all, Gene Parker has studied in some detail the interaction between small flux tubes in a whole series of papers. Secondly, some calculations on magnetoconvection suggest, although in a rather uncertain way, that in a system of magnetic fields in the presence of convection the favoured configuration is one where the smaller flux tubes are amalgamated into larger fields rather than being disseminated.

Spicer: I have examined Parker's publications and Spruit and I have discussed this and parallel currents are not considered.

Weiss: Well, I think that parallel currents are irrelevant myself.

Spicer: But they are attractive. They are the reason for a coalescence instability. 
Weiss: They are irrelevant in the atmosphere, I believe, but not in the interior.

Venugopal: How does one explain the Evershed Effect i.e. the outflow of gases.

Mullan: The Evershed Effect is a surface cellular effect in which outflow occurs at the photospheric level and inflow occurs at the chromospheric level. That probably involves Lorentz forces which are going on in the higher levels of the atmosphere and which I have not considered here. The calculations of the convective models which I have done begin in the convective zone. I believe that there is an array of surface dynamic phenomena which are occuring in the surface layers and which include the Evershed Effect which I have not dealt with here. If you want a very important example I can only refer to the very important survey which Ron Moore has carried out recently. But I agree that the surfaces of spots are the sites of very interesting dynamic phenomena.

Rodonò: I think we have very clear observational evidence in the stellar case of the connection between spots and flares. In fact the most energetic flares are, on average, observed in the more luminous stars i.e. the $\mathrm{K}$ stars and these are also those showing spots or at least exhibiting phenomena which can be interpreted as spots.

van Leeuwen: I would object to that. We have observations too of those stars which got ... (rest of comment lost) ... changes in brightness and colour that the total integrated brightness remained constant. So we have an indication that for these stars we have a redistribution of flux rather than flux storage with later release.

Rodonò: I would like to refer to the IAU Colloquium in 1974 in Moscow where a plot was presented which clearly shows what I mean. 